\title{
Quasi-ballistic and superdiffusive transport for impulsive solar particle events
}

\author{
E. M. Trotta and G. Zimbardo
}

\author{
Department of Physics, University of Calabria, Rende, Italy \\ e-mail: etrotta@thematica.it; zimbardo@fis.unical.it
}

Received 7 December 2010 / Accepted 10 March 2011

\begin{abstract}
Context. The propagation of solar energetic particles encompasses a number of transport regimes, which goes from diffusive transport to scatter-free propagation. On the other hand, numerical simulations in the presence of magnetic turbulence, as well as the analysis of propagating particles accelerated at interplanetary shocks, show that superdiffusive regimes, which are intermediate between scatter free and diffusive propagation, can be found.

Aims. In this work we study both proton and electron transport in order to understand whether both superdiffusive and ballistic propagation are indeed possible, at variance with the standard paradigm.

Methods. We carry out an analysis of impulsive solar energetic particles (SEPs) events, for which the observed time profile of energetic particle fluxes represents the propagator of the corresponding transport equation. Time profiles are fitted by power laws. Assuming well-known forms of the particle propagator, with power-law asymptotic behaviour, we determine the transport regime of particle propagation from the time profiles.

Results. Using data obtained from ACE and SoHO spacecraft, several proton and electron events that exhibit both superdiffusive and ballistic transport will be shown. When these anomalous regimes are found, no finite mean free path can be defined.
\end{abstract}

Key words. Sun: corona - Sun: particle emission - diffusion - solar wind - turbulence - Sun: flares

\section{Introduction}

The solar corona is a powerful particle accelerator, because it is able to accelerate ions to energies of the order of $1 \mathrm{GeV}$, and electrons to energies of tens of $\mathrm{MeV}$. These particles escape the coronal plasma and propagate in the solar wind along the spiral magnetic field. Understanding the propagation of energetic particles accelerated at solar flares, coronal mass ejections (CMEs), and interplanetary shocks in the presence of a turbulent magnetic field is one of the main objectives of plasma astrophysics. Indeed the transport properties are crucial for assessing shock acceleration mechanisms (e.g., Duffy et al. 1995; Sandroos \& Vainio 2009), for interpreting extreme UV observation of filaments in coronal loops (e.g., Galloway et al. 2006; Bitane et al. 2010) and in coronal hole jets (e.g. Nisticó et al. 2009), and for forecasting the arrival of harmful energetic electrons and protons accelerated near the sun on Earth (Dalla et al. 2003; Ippolito et al. 2005). Because of a background magnetic field $B_{0}$, parallel transport and perpendicular transport can have different properties, owing to particle gyromotion, as particles gyrate in the plane perpendicular to the magnetic field and move "freely" along the magnetic field. However, in the presence of magnetic fluctuations, magnetic field lines are distorted, giving rise to a field line random walk (e.g., Shalchi \& Kourakis 2007a). In addition, resonant wave particle interactions cause pitch-angle diffusion and decoupling of particles from field lines (e.g., Hauff et al. 2010).

For the turbulence levels typical of the solar wind, $\delta B / B_{0} \sim$ $0.5-1$, several transport regimes have been considered for the parallel transport of solar energetic particles (SEPs): scatter-free propagation is usually found for electrons (Lin 1974), while proton propagation is often assumed to be diffusive, $\left\langle\Delta z^{2}\right\rangle=$ $2 D_{\|} t$ (e.g., Giacalone \& Jokipii 1999; Teufel \& Schlickeiser 2002; Zhang et al. 2003). However study of many proton events, based on the onset time analysis, shows that scatter free, i.e. ballistic, transport is also possible for first arriving protons (Krucker \& Lin 2000; Dalla et al. 2003). We note that diffusive transport in the direction parallel to the average magnetic field is described by a diffusion coefficient $D_{\|}$, and is associated to a parallel mean free path given by $\lambda_{\|}=3 D_{\|} / v$, where $v$ is the particle speed. The value of $\lambda_{\|}$is very important in the theory of diffusive shock acceleration; however, its value, even for protons propagating from the Sun to the Earth, is not well known, with estimate that range from about 0.01 AU to several AU (e.g., Reames 1999; Teufel $\&$ Schlickeiser 2002; Chollet et al. 2010). The large variability of the inferred values of $\lambda_{\|}$may reflect that diffusive transport is not the only possibility for parallel propagation in the solar wind. In other words, besides diffusive transport and scatter-free (i.e. ballistic) propagation, other transport regimes can be found, in which the particle mean square deviation grows nonlinearly with time, that is, $\left\langle\Delta z^{2}\right\rangle \sim t^{\alpha}$.

When $\alpha<1$, we have subdiffusion, and we have super diffusion when $\alpha>1$. Recently, Perri \& Zimbardo (2009a) have shown that energetic ions accelerated at the solar wind termination shock propagate superdiffusively, with $\left\langle\Delta z^{2}\right\rangle=2 D_{\|}^{\alpha} t^{\alpha}$ and $\alpha \simeq 1.3$, with $\Delta z$ the field line length upstream of the shock. A parallel superdiffusive behaviour is the result of weak pitch-angle diffusion, so that the particle parallel velocity does not change sign very frequently, and very long parallel displacements, called Lévy flights, happen. Weak pitch-angle diffusion can came from low turbulence levels or from the wave 
particle resonance condition being difficult to satisfy. At the same time, parallel motion is not completely scatter-free, so that an intermediate, superdiffusive transport results. It is interesting to notice that several, independent numerical simulations of particle transport in the presence of magnetic turbulence have shown that, depending on the simulation parameters, perpendicular transport can be subdiffusive (Qin et al. 2002a; Zimbardo et al. 2006; Shalchi \& Kourakis 2007b), while parallel transport can be superdiffusive (e.g., Zimbardo et al. 2006; Gkioulidou et al. 2007; Shalchi \& Kourakis 2007b; Tautz 2010; Pommois et al. 2007). These recent result are therefore at variance with the traditional results of the so-called quasi linear theory (Giacalone \& Jokipii 1999; Teufel \& Schlickeiser 2002), which predicts normal diffusion both parallel and perpendicular to the average magnetic field.

In this paper we analyse a number of impulsive solar energetic electron and proton events, by studying the temporal profile of the particle fluxes in order to determine the transport regime. Different transport regimes are characterized by different propagators (Ragot \& Kirk 1997; Webb et al. 2006; Perri \& Zimbardo $2007,2008 a, b)$, and we show that the observed energetic particle time profiles correspond to quasi-ballistic and superdiffusive regimes.

\section{Propagators for non Gaussian transport}

The method that we use is based on the propagator formalism. The propagator $P\left(\boldsymbol{r}-\boldsymbol{r}^{\prime}, t-t^{\prime}\right)$ is the probability density function that a particle injected in $\boldsymbol{r}^{\prime}$ at time $t^{\prime}$ is found in $\boldsymbol{r}$ at time $t$, and is obtained as the solution of the appropriate transport equation for the sharp initial conditions $P(\boldsymbol{r}, t=0)=\delta(\boldsymbol{r}) \delta(t)$ (e.g., Metzler \& Klafter 2000). For the case of particles injected in a homogeneous system at different positions and times, the number density $n(\boldsymbol{r}, t)$ can be obtained as

$n(\boldsymbol{r}, t)=\int P\left(\boldsymbol{r}-\boldsymbol{r}^{\prime}, t-t^{\prime}\right) q\left(\boldsymbol{r}^{\prime}, t^{\prime}\right) \mathrm{d}^{3} r^{\prime} \mathrm{d} t^{\prime}$

where $q\left(\boldsymbol{r}^{\prime}, t^{\prime}\right)$ is the particle number density per unit time at the injection places and times. We can assume that for each given energy $E$ the flux of particles is proportional to the number density of particles with that energy. The propagator describes the transport properties, and it can be seen that for different transport models and different particle injections at the source, a wide variety of particle densities at the observer in $\boldsymbol{r}$ can be obtained as a function of time. Let us consider a few limiting cases.

Sharp localized injection at the origin: in such a case $q\left(\boldsymbol{r}^{\prime}, t^{\prime}\right)=A \delta\left(\boldsymbol{r}^{\prime}\right) \delta\left(t^{\prime}\right)$, where $A$ is a normalization constant with appropriate physical dimensions, and the integration of Eq. (1) immediately yields

$n(\boldsymbol{r}, t)=A P(\boldsymbol{r}, t)$

in agreement with the fact that the propagator is the solution of the transport equation for $\delta$-function initial conditions.

Ballistic propagation: in such a case, particles move freely away from the source at a constant speed. We consider the onedimensional (1D) case for clarity, so that the propagator is given by Webb et al. (2006)

$P\left(z-z^{\prime}, t-t^{\prime}\right)=\frac{1}{2}\left[\delta\left(z-z^{\prime}-v\left(t-t^{\prime}\right)\right)+\delta\left(z-z^{\prime}+v\left(t-t^{\prime}\right)\right)\right]$

where the $\delta$ functions describe the propagation at constant speed in the two opposite directions. Considering a particle injection localized in space but not in time, $q\left(z^{\prime}, t^{\prime}\right)=A \delta\left(z^{\prime}\right) f\left(t^{\prime}\right)$, and inserting the above expression into Eq. (1), we obtain upon integration over $z^{\prime}$

$n(z, t)=\frac{A}{2} \int\left[\delta\left(z-v\left(t-t^{\prime}\right)\right)+\delta\left(z+v\left(t-t^{\prime}\right)\right)\right] f\left(t^{\prime}\right) \mathrm{d} t^{\prime}$

whence, assuming that the observer is at $z>0$,

$n(z, t)=\frac{A}{2|v|} f\left(t-\frac{z}{v}\right)$.

(For $t>t^{\prime}, v>0$ the second $\delta$ function is always zero.) It can be seen that, for ballistic propagation, the time profile of particle emission at the source is translated to the observer in $z$ undistorted and backshifted in time by a lag $z / v$ - the time of flight. If, on the other hand, the transport is not purely ballistic, the time profile at emission $f\left(t^{\prime}\right)$ will be modified by propagation (for instance, it will be smoothed).

Particle emission at a traveling planar shock: we assume that the observer-shock distance is much less than the size of the shock, as in the case of interplanetary shocks. In such a case the particle source can be assumed to be an infinite plane, say the $x y$ plane, which moves along the $z$ direction with velocity $V_{\mathrm{sh}}$. The problem is then $1 \mathrm{D}$, and the source of particles can be described as $q\left(z^{\prime}, t^{\prime}\right)=A \delta\left(z^{\prime}-V_{\mathrm{sh}} t^{\prime}\right)$, so that the particle number density at the observer will be

$n(z, t)=A \int P\left(z-z^{\prime}, t-t^{\prime}\right) \delta\left(z^{\prime}-V_{\mathrm{sh}} t^{\prime}\right) \mathrm{d} z^{\prime} \mathrm{d} t^{\prime}$.

This is the case considered by Perri \& Zimbardo (2007, 2008a) for analysis of energetic particles accelerated at corotating interaction region (CIR) shocks, where the scale size of the CIR shock, about 5-10 AU, is much greater than the spacecraftCIR distance, below 1 AU for the considered shocks. Perri \& Zimbardo $(2007,2008$ a) used the above expression to show that, if a Gaussian propagator is assumed, corresponding to normal diffusion, an exponential profile $\exp \left(-V_{\mathrm{sh}} z / D\right)$ is obtained for the accelerated particle fluxes, in agreement with the classical view (e.g., Lee \& Fisk 1982). Conversely, if a power-law propagator is assumed, corresponding to superdiffusion, a power-law particle profile is obtained.

In the following, we analyse the observed particle profiles for a number of SEP events. The particle time profiles are fitted by power laws: considering propagators with asymptotic time profiles, the transport regime for the particles is determined from the fit. To proceed, we make the following assumptions:

1. localized injection of energetic particles, described by $\delta(\boldsymbol{r}) \delta(t)$, so that Eq. (2) holds. In such a case, the observed particle profile corresponds to the propagator. This restricts our analysis to impulsive SEP events, where energetic particles are injected at once in a localized region, probably by a flare;

2. no multiple injections of energetic particles for the same event. This requires a careful selection of the analysed events;

3. normal perpendicular transport, i.e., diffusive, so that the propagator for perpendicular transport has the well known Gaussian form. Therefore, we only investigate the possibility of anomalous transport for propagation along the magnetic field.

We now consider a localized injection of energetic particles at time $t^{\prime}=0$ and position $\boldsymbol{r}^{\prime}=(0,0,0)$. The transport properties 
can be described by the probability $P(\boldsymbol{r}, t)$ of observing the injected particles at $\boldsymbol{r}=(x, y, z)$ at time $t$. For normal diffusion in the presence of a magnetic field, this can be written as (e.g., Ragot \& Kirk 1997; Metzler \& Klafter 2000; Webb et al. 2006)

$P(\boldsymbol{r}, t)=\frac{N_{0}}{(4 \pi t)^{3 / 2} \sqrt{D_{\perp}^{2} D_{\|}}} \exp \left[-\frac{x^{2}+y^{2}}{4 D_{\perp} t}-\frac{z^{2}}{4 D_{\|} t}\right]$

where $N_{0}$ is the total number of injected particles, while $D_{\perp}$ and $D_{\|}$represent the diffusion coefficients perpendicular and parallel to the average magnetic field $\boldsymbol{B}_{\mathbf{0}}$, respectively, with $\boldsymbol{B}_{\mathbf{0}}=$ $B_{0} \hat{e}_{z}$. For magnetic turbulence levels $\delta B / B_{0} \sim 0.5-1.0$, typical of the solar wind, $D_{\|}$is usually much larger than $D_{\perp}$ (Giacalone \& Jokipii 1999; Pommois et al. 2007). In what follows, we will select impulsive SEP events, which approximate the localized injection well at the origin of coordinates. In such a case the observed particle flux is proportional to the propagator, which describes the space-time distribution of the particles injected at the origin of space and time. In our analysis, we consider the observed particle fluxes for late times, that is, for times such that the absolute value of the argument of the exponential functions in Eq. (7) is much less then 1, e.g. $z^{2} / 4 D_{\|} t \ll 1$. In such a case, the exponential functions tend to $\lim _{x \rightarrow 0} \exp \left[-x^{2}\right]=1$, and the long time behaviour of the particle flux is given by the factor

$$
\frac{N_{0}}{(4 \pi t)^{3 / 2} \sqrt{D_{\perp}^{2} D_{\|}}} \text {. }
$$

Therefore, a power law decay of the energetic particle time profile proportional to $t^{-3 / 2}$ is indicative of normal Gaussian diffusion.

On the other hand, in the case of anomalous, superdiffusive transport, the propagator can be obtained in the framework of continuous time random walks (Klafter et al. 1987; Zumofen \& Klafter 1993; Friedrich et al. 2006) in Fourier-Laplace space. Superdiffusion is characterized by a space-time, coupled probability of jumps by the random walker of the form $\psi(l, \tau)=$ $C l^{-\mu} \delta(l-v \tau)$, which is valid for large $l$, where $l$ is the jump length, $v$ the walker velocity, and $\tau$ the jump time. Such a probability defines a Levy random walk whose distinctive property is a power-law distribution of jump lengths with exponent $\mu$ (Klafter et al. 1987), and should not be confused with the propagator itself. The power-law distribution of jump (i.e., free displacement) lengths means that long jumps have a small but non-negligible probability. These long jumps make transport faster than normal, and long jumps are frequent enough for $2<\mu<3$ to enforce superdiffusion. It is important to notice that the mean square value of $l$ is diverging for $\mu<3$, so that no mean free path can be defined, and the central limit theorem, which would imply normal diffusion, does not apply. The power-law distribution of jump lengths in the Lévy random walk implies (by means of a proper statistical treatment) a power-law probability density function for the random walker position, or, in other words, a propagator with power law tails (Zumofen \& Klafter 1993). For a full discussion, see the reviews by Bouchaud (1990); Klafter et al. (1996); Metzler \& Klafter $(2000,2004)$. Therefore, while normal diffusion is related to a Gaussian propagator and, usually, a Gaussian distribution of jump lengths, which corresponds to Brownian motion, anomalous diffusion is related to propagators and distribution of jump lengths having power-law tails.

We then consider superdiffusion along the $z$ direction, which is the direction of the average magnetic field, while perpendicular transport here is assumed to be normal. In a few limiting cases, the Fourier-Laplace transform of the propagator can be inverted

1. for long times (near the source), that is for $|z| \ll k_{\mu}^{\frac{1}{2}} t^{1 /(\mu-1)}$, we have

$$
P(z, t)=a_{0} t^{1 /(1-\mu)} \exp \left[-\frac{z^{2}}{k_{\mu} t^{2 /(\mu-1)}}\right] ;
$$

2. for short times (far from the source), that is for $|z| \gg$ $k_{\mu}^{\frac{1}{2}} t^{1 /(\mu-1)}$, we have for $z<v t$

$$
P(z, t)=b \frac{t}{z^{\mu}}
$$

and $P(z, t)=0$ for $z>v t$, where $a_{0}, b$, and $\mu$ are constants and $k_{\mu}$ is an anomalous diffusion constant (Zumofen \& Klafter 1993). The value of $\mu$ determines the type of transport: superdiffusion is obtained for $2<\mu<3$, transport is diffusive for $3<\mu<4$ even if the propagator is non-Gaussian but a power law, and transport is ballistic (i.e. scatter-free) for $1<\mu<2$ (Zumofen \& Klafter 1993). Superballistic regimes can also be obtained, see Klafter et al. (1987) and Zimbardo et al. (2000). For $2<\mu<3$, the following diffusion law is valid:

$$
\left\langle\Delta z^{2}(t)\right\rangle=2 D_{\alpha} t^{\alpha}
$$

where the exponent of anomalous diffusion $\alpha$ is related to the exponent $\mu$ in the propagator by $\alpha=4-\mu$. In our analysis of impulsive SEP events, the propagator form related to superdiffusive transport is used, in particular, its form calculated in real space in the approximation of long times (i.e. $|z| \ll k_{\mu}^{\frac{1}{2}} t^{1 /(\mu-1)}$ ). In such a case the exponential term of (9) stretches to 1, and the propagator assumes the form

$P(z, t) \sim \frac{a_{0}}{t^{1 /(\mu-1)}}$.

We note that the comparison between power-law propagators and tracer particle distributions is also used in laboratory plasmas to determine the transport regimes (del Castillo-Negrete et al. 2005).

We now make the assumption that perpendicular transport is normal in most cases: although subdiffusive and sometimes superdiffusive transport have been found for perpendicular transport (Qin et al. 2002a; Zimbardo 2005; Zimbardo et al. 2006; Shalchi \& Kourakis 2007b), numerical simulations show that perpendicular transport is normal in most cases of strong turbulence (Pommois et al. 2007; Tautz 2010). In particular, Qin et al. (2002a) show that perpendicular subdiffusion is obtained in the case of a prevailing slab turbulence, while Qin et al. (2002b) show that normal diffusion is recovered in the case of prevailing two dimensional turbulence, as may occurs in the solar wind. Also, perpendicular subdiffusion is usually the result of particles tracing back the magnetic field lines, a phenomenon called compound diffusion; however, this does not happen if enough field line stochasticity is present. Recently Zimbardo et al. (2009) and Bitane et al. (2010) have shown that field line stochasticity grows with the quasi-2D anisotropy and that perpendicular transport is usually diffusive in space plasmas. Therefore, we assume that, even in the case of parallel superdiffusion, perpendicular diffusion is described by the Gaussian propagator, that the 3-D propagator is the product of the propagators for perpendicular transport $P_{\perp}(x, y, t)$ and that for parallel transport $P_{\|}(z, t)$

$P(\boldsymbol{r}, t)=P_{\perp}(x, y, t) \times P_{\|}(z, t)$. 
We notice that this expression implies that the probabilty for perpendicular transport is independent of the probability for parallel transport; this may not be the case in a magnetized plasma due to compound diffusion, (see, Zimbardo 2005; Webb et al. 2006; Zimbardo et al. 2009). However, once the transport regime is known, the probability of being at a given position can be described in the same way as above, the probability being a statistical quantity. More in detail, we have

$$
P(\boldsymbol{r}, t)=\frac{N_{0}}{4 \pi t D_{\perp}} \exp \left[-\frac{x^{2}+y^{2}}{4 D_{\perp} t}\right] \times \frac{a_{0}}{t^{1 /(\mu-1)}} \exp \left[-\frac{z^{2}}{k_{\mu} t^{2 /(\mu-1)}}\right] .
$$

Again considering the limit for long times, we are left with

$P(\boldsymbol{r}, t) \simeq \frac{N_{0} a_{0}}{4 \pi D_{\perp} t^{1+(1 /(\mu-1))}} \sim \frac{1}{t^{\mu /(\mu-1)}}=t^{-m}$

where $m=\mu /(\mu-1)$. We have super diffusion for $2<\mu<3$, while ballistic regimes where $\left\langle(\Delta z)^{2}\right\rangle \sim v^{2} t^{2}$ are found for $\mu<2$ (Zumofen \& Klafter 1993), and normal diffusion is found for $\mu>3$. Therefore, the slope of the particle flux decay for long times should be $m=3 / 2$ for normal diffusion, $m=2$ for nearly ballistic transport, and $3 / 2<m<2$ for superdiffusive transport. In other words, a slope of the particles flux decay greater than $3 / 2$ is indicative of either superdiffusive or ballistic transport. Also, for $3 / 2 \leq m \leq 2$, we obtain $\mu=m /(m-1)$, and the anomalous diffusion exponent is $\alpha=4-m /(m-1)$.

We note that, in the case of strictly ballistic transport, the propagator is given in terms of delta functions, as seen in the Introduction. However, Zumofen \& Klafter (1993) have shown that anomalous quasi-ballistic transport with some scattering, which may correspond more closely to the observed scatter-free events, may also be described in terms of CTRW, provided the exponent $\mu$ in the jump probability $\psi(l, \tau)=C l^{-\mu} \delta(l-v \tau)$ satisfies $1<\mu<2$. In such a case, particles are moving back and forth, but long free displacement have such a high probability that $\left\langle(\Delta z)^{2}\right\rangle \sim v^{2} t^{2}$ results (Klafter et al. 1987; Zumofen \& Klafter 1993). For $1<\mu<2$, the analytical inversion of the FourierLaplace transform is not known in general; that is, Eqs. $(9,10)$ do not hold. However, we suggest that, when $m=\mu /(\mu-1)>2$, this may correspond to $\mu<2$, that is, to quasi-ballistic transport, which, including some scattering, may be a more realistic and complete description of scatter-free events. In particular, Perri \& Zimbardo (2008b) suggest that the spike in the propagator obtained for $\mu=3 / 2$ corresponds to the observed electron profiles for scatter-free events. Finally, other forms of the propagator for non Gaussian transport have been given by Ragot \& Kirk (1997) and by Webb et al. (2006).

\section{Data analysis}

In this section we describe the events we analysed, showing the results for the transport properties and the criteria used for selecting the events. We selected SEPs observations to make it possible to assume that the energetic particles are accelerated in an impulsive event like a flare. This is a necessary condition for comparing the observed particle flux to the propagator, since the propagator describes the evolution of the particle distribution in the case of injection localized in space and time. Therefore, SEPs accelerated at CMEs are not suitable for the present analysis. In particular, for particles continuously accelerated at a traveling shock, as in the case of corotating interaction regions, a different approach was developed (Perri \& Zimbardo 2007, 2008a, 2009b). The events reported here were chosen by analyzing SoHO and ACE observations in 2000-2002, close to the solar maximum in order to have more impulsive SEPs events. Regarding the SoHO spacecraft, we used SoHO Comprehensive Suprathermal and Energetic Particle Analyzer (COSTEP - Müller-Mellin et al. 1995). These data are relative to 5 minutes average proton and electron fluxes. In particular we used

- proton fluxes measured by SoHO - CST in 2 energy channel: 16.40 MeV and 33.0 MeV.

Concerning the ACE spacecraft, we used ACE/EPAM (Electron Proton Alpha Monitor) data to 1 -h averaged proton and electron fluxes. In particular we used

- proton fluxes measured by ACE/EPAM in four energy channels: $0.112-0.187 \mathrm{MeV}, 0.187-0.310 \mathrm{MeV}, 0.310-$ $0.580 \mathrm{MeV}$, and $0.580-1.060 \mathrm{MeV}$;

- the electron fluxes measured by ACE/EPAM four energy channels: 0.038-.053 MeV, 0.053-0.103 MeV, 0.103$0.175 \mathrm{MeV}, 0.175-0.315 \mathrm{MeV}$.

\subsection{Event selection criteria}

All the dataset we consider were obtained from the CDAWeb service of the National Space Science Data Center (cdaweb.gsfc. nasa.gov). The selection of the events starts with identifying those periods with no influence on transient events like CMEs, so that the background conditions remained relatively unperturbed and the event profiles are very well defined and easily recognized. The characterization of the impulsive events follows the criteria proposed by Reames (1999): intensity-time profiles of electrons and protons with a prevalence of electron intensity and a duration of tens of hours. Besides this, they are ${ }^{3} \mathrm{He}$-rich events. Normally impulsive events came from a flare (or a series of flares) without associated CME (pure event). Event selection starts from examining electron fluxes from ACE/EPAM datasets. Events that exhibit a characteristic intensity-time profile, i.e. fast rise and smooth decay, were selected for ${ }^{3} \mathrm{He}$ analysis. The ${ }^{3} \mathrm{He}$ data are from ACE/ULEIS (Ultra Low Energy Isotope Spectrometer) instrument and are related to the same time interval of the chosen event: only ${ }^{3} \mathrm{He}$-rich event were chosen. Impulsive SEPs event are also characterize by the short $[<1 \mathrm{~h}]$ duration of the associated soft X-ray (SXR) burst. To test the SXR burst duration we inspected data from the GOES-8 Space Environment Monitor for the same time interval. We analysed the time profile in the long time approximation looking for those events that exhibit a superdiffusive (ballistic) appearance either for proton and/or for electron flux.

To analyse the characteristic of intensity-time profiles (e.g. the diffusion properties), we inspected each event to determine the origin in the corona, i.e. the start time of the event. To do that we looked for a type III radio burst related to impulsive SEPs event. Type III radio burst data are from WIND Waves RAD2. The occurrence of radio type III bursts, starting in the corona and traveling into interplanetary space, are related to the flare-accelerated electrons at the origin of the event. The analysis was made using 10 minute averages and time-intensity graphics. Assuming that the spacecraft is connected well to the flare site, the event's start time coincides with the type III onset time (e.g. the electron release coincides with radio type III burst (see also Krucker \& Lin 2000)).

Many studies have focused on the role of the heliographic longitude of the source by analyzing SEPs data from near-earth spacecraft (Reames 1999; Krucker \& Lin 2000; Dalla et al. 2003; Ippolito et al. 2005). Those studies find that impulsive 
Table 1. Main parameters of selected event.

\begin{tabular}{lcccccccccc}
\hline \hline Data & $\begin{array}{l}E_{\max } \\
\mathrm{MeV}\end{array}$ & Satellite & $\begin{array}{c}t_{0} \\
h\end{array}$ & $\begin{array}{c}t_{1} \\
h\end{array}$ & $\begin{array}{c}t_{\max } \\
h\end{array}$ & $\begin{array}{c}m \\
h\end{array}$ & $\alpha$ & $\mu$ & $\chi^{2}$ & Flare-coords \\
\hline 2000 June 10 & & & & & & & & & & \\
Protons & 16.40 & SoHO & $17: 00$ & 1.00 & 2.00 & 2.09 & 2.00 & 1.92 & 0.20 & N22W38 \\
& 33.00 & SoHO & $17: 00$ & 2.00 & 2.00 & 2.19 & 2.00 & 1.84 & 0.22 & \\
\hline Electrons & 0.05 & ACE & 17.00 & 1,00 & 1,00 & 1.56 & 1.22 & 2.78 & 0.36 & N22W38 \\
& 0.10 & ACE & 17.00 & 1.00 & 1.00 & 1.69 & 1.56 & 2.44 & 0.24 & \\
& 0.18 & ACE & 17.00 & 1.00 & 1.00 & 1.76 & 1.69 & 2.31 & 0.12 & \\
& 0.32 & ACE & 17.00 & 1.00 & 1.00 & 1.79 & 1.73 & 2.27 & 0.09 & \\
\hline 2001 July 19 & & & & & & & & & & \\
Electrons & 0.05 & ACE & $4: 00$ & 5.00 & 10.00 & 1.74 & 1.64 & 2.36 & 0.08 & N13W72 \\
& 0.10 & ACE & $4: 00$ & 5.00 & 10.00 & 1.72 & 1.61 & 2.39 & 0.02 & \\
& 0.18 & ACE & $4: 00$ & 5.00 & 10.00 & 1.61 & 1.36 & 2.64 & 0.01 & \\
\hline 2002 Feb. 20 & 0.32 & ACE & $4: 00$ & 5.00 & 10.00 & 1.23 & 1.00 & - & 0.03 & \\
Protons & 0.112 & ACE & 5.55 & 2.00 & 18.00 & 1.30 & 1.00 & - & 0.28 & N12W72 \\
& 0.187 & ACE & 5.55 & 2.00 & 18.00 & 1.11 & 1.00 & - & 0.15 & \\
& 0.310 & ACE & 5.55 & 2.00 & 18.00 & 0.99 & 1.00 & - & 0.08 & \\
& 0.580 & ACE & 5.55 & 2.00 & 18.00 & 0.97 & 1.00 & - & 0.05 & \\
\hline Electrons & 0.05 & ACE & 5.55 & 1.00 & 2.00 & 1.97 & 1.97 & 2.03 & 0.09 & N12W72 \\
& 0.10 & ACE & 5.55 & 1.00 & 2.00 & 2.12 & 2.00 & 1.89 & 0.07 & \\
& 0.18 & ACE & 5.55 & 1.00 & 2.00 & 2.16 & 2.00 & 1.87 & 0.04 & \\
& 0.32 & ACE & 5.55 & 1.00 & 2.00 & 2.03 & 2.00 & 1.97 & 0.03 & \\
\hline
\end{tabular}

Notes. $E_{\max }$ is the maximum value of the energy channel. The columns $t_{0}, t_{1}$, and $t_{\max }$ are the start time in corona, the time delay from start to instrument detector, and time delay from start to of particle-flux maximum. The columns $m, \mu$, and $\alpha$ are related to the transport regime, see text. The column $\chi^{2}$ shows the value of the $\chi^{2}$ test for $m$ fit.

SEPs event can be observed when the source region is in the well-connected longitudinal range (that is, from W20 to W90). The positional data of the flare (e.g. coordinates) associated to the event is compared with intervals in the well-connected longitudinal range. The flare data are from National Geophysical Data Center (NGDC) solar data. Among the many events that have been identified we report here three events that serve to exemplify the different transport regimes. The event characteristics and the obtained transport regimes are given in Table 1 .

\subsubsection{June 10}

The 2000 June 10 impulsive event is shown in Fig. 1. This is associated with an M 5.2 class GOES flare at heliographic position N22W38. In the left column of Fig. 1 from top to bottom are shown, on a lin-log scale, the proton fluxes measured by SoHO in the energy channels $16.4 \mathrm{MeV}$ and $33.0 \mathrm{MeV}$, the electron flux from ACE/EPAM in the energy channels $0.038-0.053 \mathrm{MeV}$, 0.053-0.103 MeV, 0.103-0.175 MeV, 0.175-0.315 MeV, and the ${ }^{3} \mathrm{He}$ fluxes measured by ACE/ULEIS in the energy channels $0.072-0.160 \mathrm{MeV}, 0.160-0.320 \mathrm{MeV}, 0.320-0.640 \mathrm{MeV}$, 0.640-1.280 MeV, and the X-rays flux from GOES/SEM in the wavelengths $1-8 \AA$ (HXR) and $0.5-3 \AA$ (SXR). In the graphs, the data are averaged hourly and are synchronized with the start time at the source. The ${ }^{3} \mathrm{He}$ peak is delayed $\sim 20 \mathrm{~h}$ owing to the lower ${ }^{3} \mathrm{He}$ speed. Indeed, considering the speed as a function of energy and mass of particles and assuming that the distance traveled along the magnetic field spiral is about 1.2 AU (e.g., Krucker \& Lin 2000), we find that the ${ }^{3}$ He flux should be shifted at least $15 \mathrm{~h}$ with respect to the proton. The flare is shown by the peak present in X-rays, and is highly synchronized with the peak flux of protons and electrons. All this properties indicate that this is an impulsive event. The event start time is determined by analyzing radio type III burst in order to determine the origin of the time axis, as explained above.
The proton and electron fluxes suddenly rise simultaneously because we are using hourly averages, thus loosing the evidence of velocity dispersion for electrons of different energies (e.g., Lin 1974, 2005). At the same time, the spiky profiles themselves indicate a fast, nearly scatter-free propagation and a good magnetic connection with the acceleration site. The characteristics of the transport of SEPs are linked to form of the propagator during the decay phase, as explained in Sect. 2. To determine the power-law scaling of the energetic particle fluxes, we plot them on log-log axes (Fig. 1, right panels). The origin of the time axis is determined using the event start time, and the linear fit was performed on the decay phase of the event. To respect the condition that the propagator form for long times is valid, we fit the flux starting at about ten hours after the event start time, that is, well after the peak in the particle fluxes. The electron and proton graphs (Fig. 1 are on a time range of $\sim 50 \mathrm{~h}$ for all energy channels. The onset time (origin) was set to 2000 June 6 at 17:00. The peak of the event is seen at $\sim 4 \mathrm{~h}$ from the origin. The fit was made on 25 points after $\sim 10 \mathrm{~h}$ from the event's start time.

The quality of the fit was evaluated using the $\chi^{2}$ test which shows that the fit is usually very good. Also, visual inspection of time profiles in the right hand panels of Fig. 1 shows that the particle flux decay is represented well by power laws. The power-law index is given by $m=\mu /(\mu-1)$, see Eq. (15). The slope (reported in Table 1) and the calculation of the exponent $\mu$ indicate superdiffusive transport with $\alpha=1.56-1.79$ for electron, ballistic (e.g. scatter-free) transport, $\alpha \sim 2$ for protons. This type of transport is consistent with the rapid rise and decay in the energetic particles profile, typical of scatter-free events (e.g. Lin 1974).

\subsubsection{February 20}

This event, shown in Fig. 2, is characterized by different transport regimes for protons and electrons. As above, proton and 

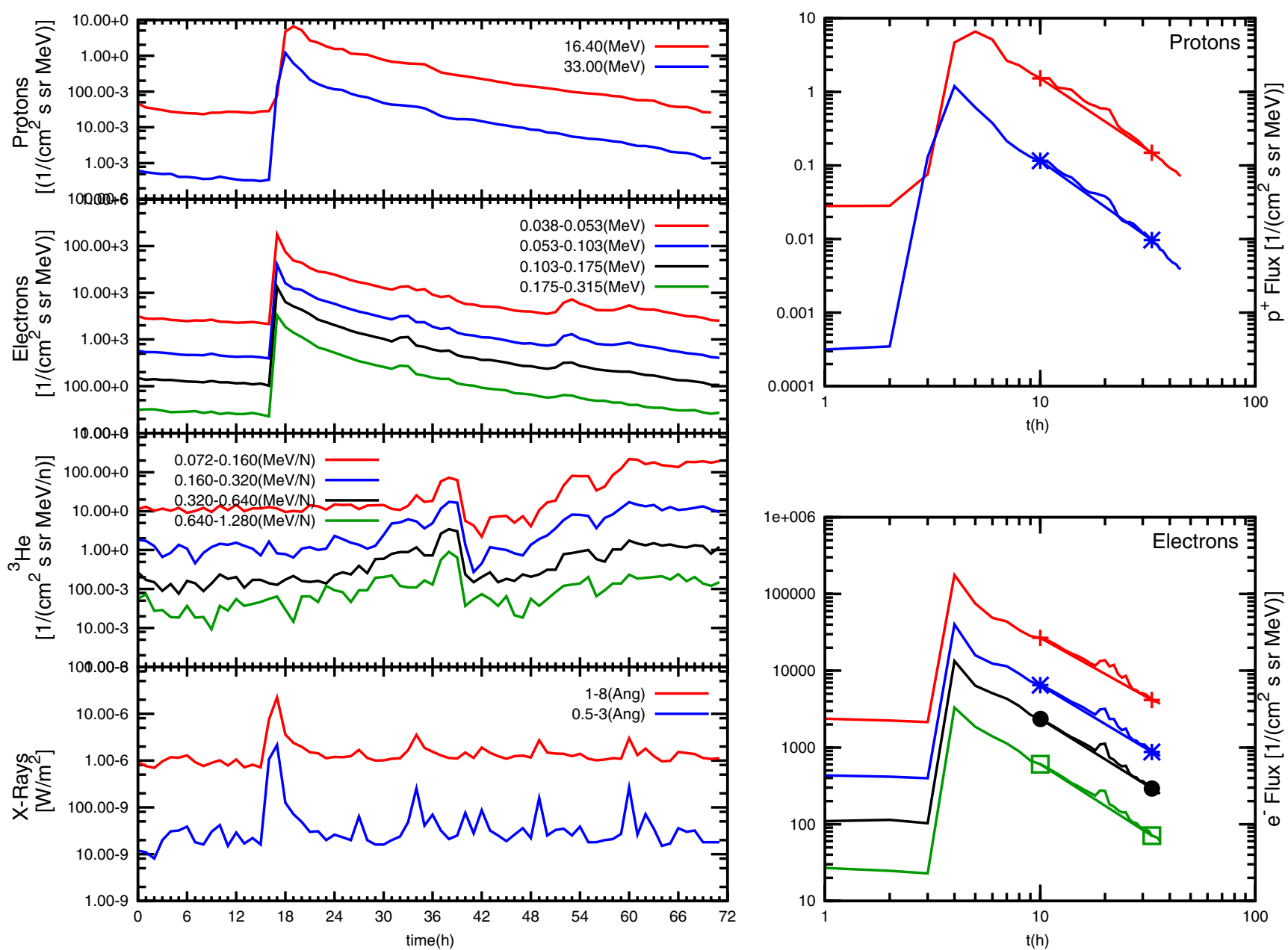

Fig. 1. 2000 June 10 impulsive event. On the left: lin-log graph of fluxes data; from top to bottom: proton flux from SoHO, electron flux from ACE/EPAM, ${ }^{3} \mathrm{He}$ flux from ACE/ULEIS and X-rays from GOES/SEM. On the right: electron and proton log-log graph with linear fit in the descending zone.

electron fluxes are compared with ${ }^{3} \mathrm{He}$ flux and SXR to characterize the event as an impulsive event. The 2002 February 20 impulsive event is associated with an M 5.1 class GOES flare at heliographic position N12W72. The prompt arrival of the electrons shows that a good magnetic connection between the spacecraft and the acceleration site is present. The start time $t_{0}$ has been set 2002 February 20 at 5:55. The peak of the event is seen at $\sim 1 \mathrm{~h}$ from $t_{0}$ for electrons and $\sim 18 \mathrm{~h}$ from $t_{0}$ for protons. This long delay of the proton maximum is due to the lower proton energies measured by ACE, $0.11-1.06 \mathrm{MeV}$, compared to those of SoHO in Fig. 2, 16.0-33.0 MeV. As with the previous event, to determine the power-law scaling of the energetic particle fluxes, we plot them in log-log axes (Fig. 2). The origin of the time axis is determined using the event start time, and the linear fit was performed on the decay of the event excluding the peak, but starting the fit at $20 \mathrm{~h}$ delay for protons and at $10 \mathrm{~h}$ for electrons.

The quality of the fit was evaluated using the $\chi^{2}$ test. The electrons and protons (Fig. 2 on the left) profiles have different time ranges: $\sim 52 \mathrm{~h}$ for all energies channels of electrons and $\sim 72 \mathrm{~h}$ for all energies channels of protons.

Because of the different intensity-time profiles between electrons and protons, the parameters of fit were different in a number of features. The slopes of the particle profile are reported in Table 1. For protons we find $m=0.97-1.3$, that is, values lower than $3 / 2$, so that normal diffusion is obtained for protons in this event. We recall that the relation $\mu=m /(m-1)$ only holds for $3 / 2<m<2$, so that the value of $\mu$ is not given in this case in Table 1. Conversely, for electrons $m=1.97-2.16$, that is, the value nearly equal to two or larger than two. This implies ballistic or quasi-ballistic transport, i.e., $\alpha=2$. We note that such a ballistic regime is consistent with the scatter-free electron propagation considered by Lin $(1974,2005)$, and the very spiky electron profiles of this event are not very different from those considered by Lin $(1974,2005)$. However, introduction of the propagators corresponding to superdiffusive transport or to quasi-ballistic transport allows the decaying part of the electron profile to be interpreted as the result of scattering of a limited number of particles and not as the effect of continued electron acceleration at the source.

\subsubsection{July 19}

Unlike the previous events, for the 2001 July 19 event we did not analyse protons because the data relative to proton fluxes were very disturbed. The event is shown in Fig. 3 where electron fluxes are compared with ${ }^{3} \mathrm{He}$ flux and SXR. The 2001 July 19 event is associated with a flare at heliographic position N13W72. From top to bottom are shown on lin-log scale the electron flux from ACE/EPAM in the energy channels $0.038-0.053 \mathrm{MeV}$, 0.053-0.103 MeV, 0.103-0.175 MeV, 0.175-0.315 MeV, and the ${ }^{3} \mathrm{He}$ fluxes measured by ACE/ULEIS in the energy channels $0.072-0.160 \mathrm{MeV}, 0.160-0.320 \mathrm{MeV}, 0.320-0.640 \mathrm{MeV}$, 

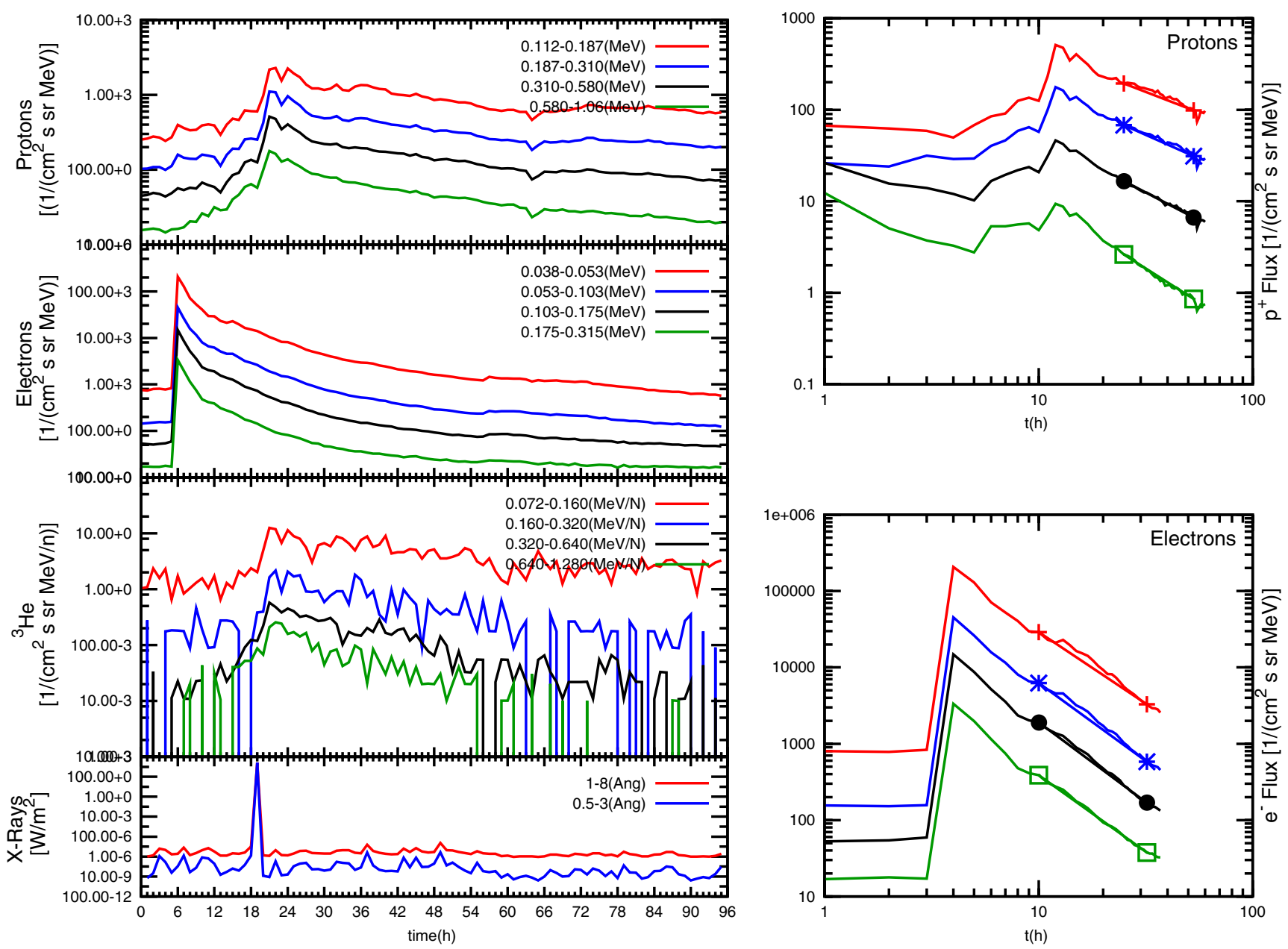

Fig. 2. Same as Fig. 1, but for the 2002 February 20 impulsive event.

$0.640-1.280 \mathrm{MeV}$. The bottom panel shows the X-ray flux from GOES/SEM in the energy channels $1-8 \AA$ (HXR) and 0.5-3 (SXR). That the magnetic connection was not good is also indicated by the electron profiles not being sharply peaked, suggesting that some cross field propagation was necessary before the electron flux maxima were reached. The flare is shown by the peak present in X-rays, and it happened about $5 \mathrm{~h}$ before the electron flux maxima.

As for the previous events, we made the power-law fit on the decay phase of the profiles, as indicated in the right panel of Fig. 3. To minimize the influence of cross field diffusion on the electron profile, we started to fit at $20 \mathrm{~h}$ after the start of the event. The power-law exponents (reported in Table 1) and the calculation of the exponent $\mu$ indicate superdiffisive transport for electrons with energies from $0.05 \mathrm{MeV}$ to $0.18 \mathrm{MeV}$, with anomalous diffusion exponent $\alpha=1.36-1.64$. On the other hand, normal diffusion is found for an electron energy of $0.175-0.315 \mathrm{MeV}$, see Table 1 . For this event, we notice that the $\alpha$ decreases with increasing energy. However, for the event of 2000 June 10, it is found that $\alpha$ increases with energy, so that a clear trend of the anomalous diffusion exponent with energy cannot be identified at this stage.

\section{Discussion and conclusions}

In this paper we have developed a method to extract information on the transport of solar energetic particles by analysing the time decay of the energetic particles fluxes measured by spacecraft in the solar wind. Our aim was to highlight the existence of superdiffusive transport regime, intermediate between normal diffusion and ballistic transport. To this end, we considered the different forms of the propagator for the different transport regime. It has been shown that for superdiffusive transport along the magnetic field, in the limit of long times, the particle flux decays as a power law with exponent $m$, where $m$ is related to $\mu$ by $\mu=m /(m-1)$, and the anomalous diffusion exponent is given by $\alpha=4-\mu$. Therefore, $3 / 2<m<2$ corresponds to superdiffusion. We analysed the time profiles of a number of impulsive SEPs and found superdiffusive and ballistic transport regimes, both for electrons and protons.

In the above analysis, we assumed that the energetic electrons and ions are injected as a $\delta$-function in space and time. From the $X$ ray peaks in Figs. 1-3, we can estimate the injection time at the source to be about 2-3 h, which is much less than the duration of the SEP event at the observer, about $50 \mathrm{~h}$. For this reason we assume that the particle emission at the source can be represented by a delta function, from which it follows that the propagator corresponds to the observed particle profile. It is interesting to notice that, if the particle injection is not a delta function, but there is a tail in the emission profile $f(t)$, this will contribute to the observed flux at late times. In this case, this means that the observed decay of the particle flux is not as steep as it would be for a delta function injection, because of the contribution of "late" particles. A steeper decay implies a higher value of $m$ (see Eq. (15)), hence higher values of $\alpha$, so we can 

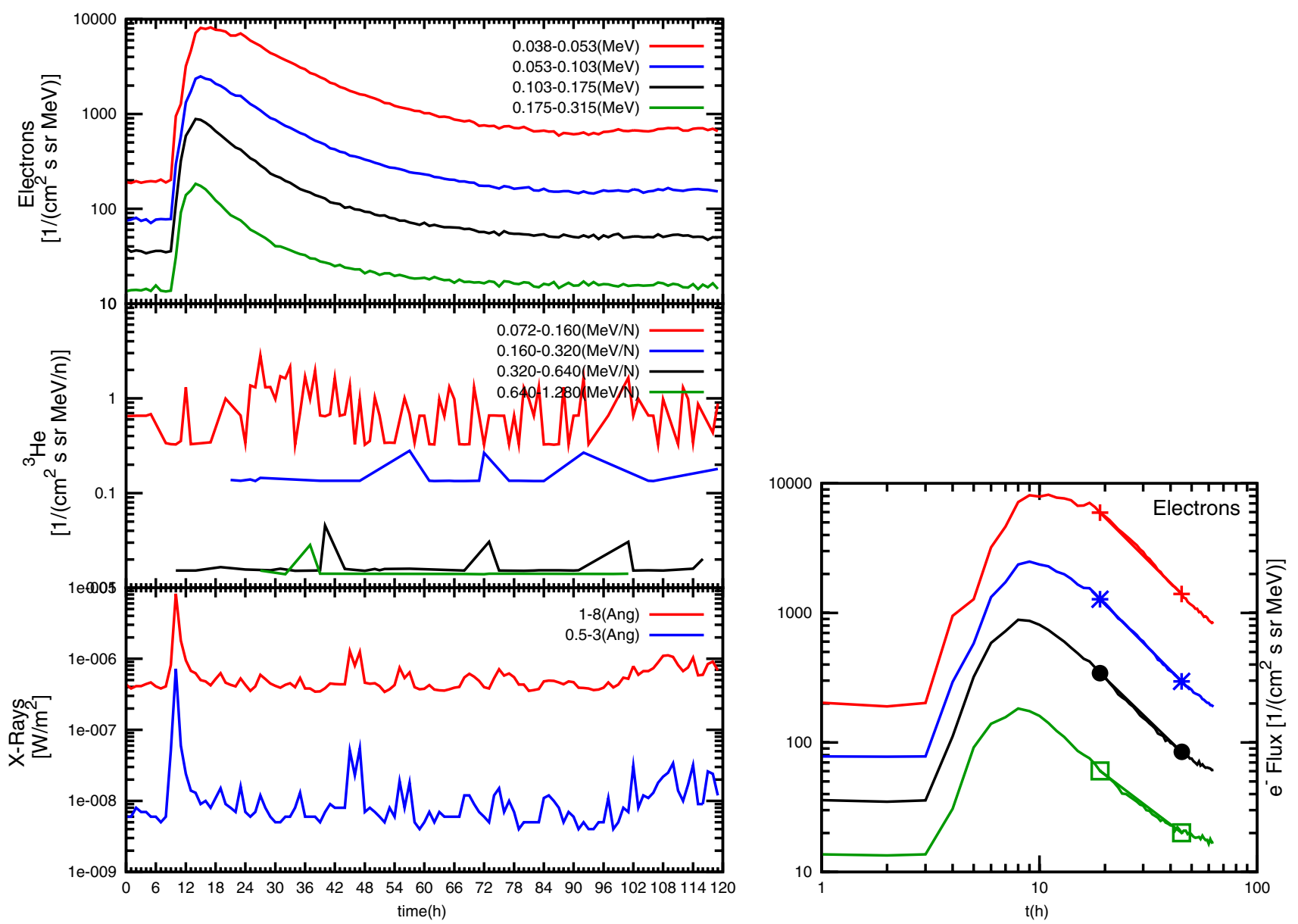

Fig. 3. 2001 July 19 impulsive event. On the left: lin-log graph of fluxes data; from top to bottom: proton flux from ACE/EPAM, ${ }^{3} \mathrm{He}$ flux from ACE/ULEIS and X-rays from GOES/SEM. On the right: electrons log-log graph with linear fit in descending zone.

say that the reported values of $\alpha$ for the superdiffusive cases are lower estimates.

For protons, we find ballistic, i.e., scatter-free, transport in one case and normal diffusion in another case. It should be noted that different proton energies were considered in those two cases, i.e., 16-33 MeV in the event of June 10, 2000, and 0.1-1.0 MeV for the event of February 20, 2002. Such different transport regimes can depend both on the particle gyroradius (e.g. Pommois et al. 2007) and on the turbulence level, and are consistent with the variety of proton mean free paths considered, for instance, by Chollet et al. (2010).

For the electrons in the energy channels from 0.01 to $0.32 \mathrm{MeV}$, we find superdiffusive transport with the exponent $\alpha$ ranging from 1.22 to 1.73 , and ballistic or quasi-ballistic transport as well. These regimes complement those found by Perri \& Zimbardo (2008a,b, 2009b), where anomalous diffusion exponent $\alpha$ from 1.1 to 1.6 were obtained, and show that a wide range of anomalous diffusion exponents $1<\alpha<2$ is possible and that superdiffusive transport can be the most frequent regime for energetic electrons. Furthermore, we also find that scatterfree transport can be interpreted in terms of the propagator and the jump probability for Levy random walk with $\mu<2$, a regime that we call quasi-ballistic. We should mention that in the above analysis we have neglected the effects of adiabatic deceleration, which leads to a decrease in time of particles with a given energy. This effect should be negligible for SEPs, as suggested by Gombosi (1979). Indeed Ruffolo (1995) finds a typical time for adiabatic deceleration of six days at $1 \mathrm{AU}$, which is much longer than the typical decay times of the analysed events. More recently Chollet et al. (2010) found by a numerical simulation that the average energy loss for a $1 \mathrm{MeV}$ proton reaching $1 \mathrm{AU}$, assuming a mean free path $\lambda=0.10-0.30 \mathrm{AU}$, is $30 \%$, which is a bit less than with the energy width of the energy channels considered above. This shows that adiabatic deceleration can be neglected in a first analysis.

The finding that both electrons and protons can exhibit a variety of transport regimes that are different from normal diffusion can be related to the fact that the magnetic turbulence level in the solar wind, which determines the amount of pitch angle scattering, actually varies considerably from event to event (see for instance the values of $\delta B / B_{0}$ reported by Ippolito et al. (2005) for several solar flares and associated SEPs events). Likewise, recent data analysis shows that magnetic turbulence in the slow solar wind, where both SoHO and ACE spend most of time, is frequently only weakly stationary (Perri \& Balogh 2010). A comparison of the obtained transport regime with the observed turbulence properties would be interesting, but a spacecraft can measure the fluctuation transported by the solar wind in the radial direction, while the particles propagate along the Parker spiral, so that a direct comparison is not easy.

The obtained results imply that, beside diffusive transport and scatter-free transport, superdiffusive transport is also possible for electrons accelerated at impulsive SEPs event. This finding is obtained from the analysis of experimental data, and 
it confirms the predictions of superdiffusive or nearly ballistic transport of numerical simulations as reported by (Zimbardo et al. 2006; Pommois et al. 2007; Shalchi \& Kourakis 2007b; Tautz 2010). Therefore a substantial rethinking of SEPs propagation is necessary, because it can influence both the acceleration mechanism and the predictions of electrons and protons fluxes required by space weather reports and the assessment of the amount of total energy required to accelerate particles at impulsive events, as determined from the observed particle fluxes.

Acknowledgements. This work was supported in part by ASI grant No. I/015/ 07/0 "Esplorazione del Sistema Solare".

\section{References}

Bitane, R., Zimbardo, G., \& Veltri, P. 2010, ApJ, 719, 1912

Bouchaud, J.-Ph., \& Georges, A. 1990, Phys. Rep., 195, Issue 4

Chollet, E., Giacalone, J., \& Mewaldt, R. 2010, J. Geophys. Res., 115, A06101

Dalla, S., Balogh, A., \& Krucker, S. 2003, Annales Geophys., 21, 1367

del Castillo-Negrete, D., Carreras, B., \& Lynch, V. 2005, Phys. Rev. Lett., 94, 065003

Duffy, P., Kirk, J., Gallant, Y., \& Dendy, R. 1995, A\&A, 302, L21

Friedrich, R., Jenko, F., Baule, A., \& Eule, S. 2006, Phys. Rev. Lett., 96, 230601

Galloway, R., Helander, P., \& MacKinnon, A. 2006, ApJ, 646, 615

Giacalone, J., \& Jokipii, J. 1999, ApJ, 520, 204

Gkioulidou, M., Zimbardo, G., Pommois, P., Veltri, P., \& Vlahos, L. 2007, A\&A, 462,1113

Gombosi, T. 1979, Physics of the Space Environment (Cambridge: Cambridge University Press)

Hauff, T., Jenko, F., Shalchi, A., \& Schlickeiser, B. 2010, ApJ, 711, 997

Ippolito, A., Pommois, P., Zimbardo, G., \& Veltri, P. 2005, A\&A, 438, 715

Klafter, J., Blumen, A., \& Shlesinger, M. 1987, Phys. Rev. A, 35, 3081
Klafter, J., Shlesinger, M., \& Zumofen, G. 1996, Phys. Today, 49, 33

Krucker, S., \& Lin, R. 2000, ApJ, 542, L61

Lee, M., \& Fisk, L. 1982, Space Sci. Rev., 32, 205

Lin, R. 1974, Space Sci. Rev., 16, 189

Lin, R. 2005, Adv. Space Res., 35, 1857

Metzler, R., \& Klafter, J. 2000, Phys. Rep., 339, 1

Metzler, R., \& Klafter, J. 2004, J. Phys. A: Math. Gen., 37, R161

Müller-Mellin, R., Kunow, H., Fleißner, V., et al. 1995, Sol. Phys., 162, 483

Nisticó, G., Bothmer, V., Patsourakos, S., \& Zimbardo, G. 2009, Sol. Phys., 259, 87

Perri, S., \& Balogh, A. 2010, ApJ, 714, 937

Perri, S., \& Zimbardo, G. 2007, ApJ, 671, L177

Perri, S., \& Zimbardo, G. 2008a, J. Geophys. Res., 113, A03107

Perri, S., \& Zimbardo, G. 2008b, Astrophys. Space Sci. Trans. (ASTRA), 4, 27

Perri, S., \& Zimbardo, G. 2009a, ApJ, 693, L118

Perri, S., \& Zimbardo, G. 2009b, Adv. Space Res., 44, 465

Pommois, P., Zimbardo, G., \& Veltri, P. 2007, Phys. Plasmas, 14, 012311

Qin, G., Matthaeus, W., \& Bieber, J. 2002a, Geophys. Res. Lett., 29, 1048

Qin, G., Matthaeus, W., \& Bieber, J. 2002b, ApJ, 578, L117

Ragot, B., \& Kirk, J. 1997, A\&A, 327, 432

Reames, D. 1999, Space Sci. Rev., 90, 413

Ruffolo, D. 1995, ApJ, 442, 861

Sandroos, A., \& Vainio, R. 2009, A\&A, 507, L2

Shalchi, A., \& Kourakis, I. 2007a, Phys. Plasmas, 14, 112901

Shalchi, A., \& Kourakis, I. 2007b, A\&A, 470, 405

Tautz, R. 2010, Plasma Phys. Control. Fusion, 52, 045016

Teufel, A., \& Schlickeiser, R. 2002, A\&A, 393, 703

Webb, G., Zank, G., Kaghashvili, E., \& le Roux, J. 2006, ApJ, 651, 211

Zhang, M., McKibben, R., Lopate, C., et al. 2003, J. Geophys. Res., 108, 1154 Zimbardo, G. 2005, Plasma Phys. Control. Fusion, 47, B755

Zimbardo, G., Greco, A., \& Veltri, P. 2000, Phys. Plasma, 443, 225

Zimbardo, G., Pommois, P., \& Veltri, P. 2006, ApJ, 639, L91

Zimbardo, G., Bitane, R., Pommois, P., \& Veltri, P. 2009, Plasma Phys. Control. Fusion, 51, 015005

Zumofen, G., \& Klafter, J. 1993, Phys. Rev. E, 47, 2 